[5] S. Sunder and V. Ramachandran, "Design of equiripple nonrecursive digital differentiators and Hilbert transformers using weighted least-squares technique," IEEE Trans. Signal Processing, pp. 2504-2509, Sept. 1994

[6] K. P. Pun, J. E. Franca, and C. A. Leme, "Polyphase SC IIR Hilbert transformer," Electron. Lett., vol. 35, pp. 689-690, Apr. 1999.

[7] A. Hiroi, K. Endo, H. Kamata, and Y. Ishida, "Design and implementation of an ideal Hilbert transformer using neural networks," in Proc. IEEE Pacific Rim Conf. Communication, Computer and Signal Processing, vol. I, 1993, pp. 292-295

[8] S. Samadi, Y. Igarashi, and H. Iwakura, "Design and multiplierless realization of maximally flat FIR digital Hilbert transformers," IEEE Trans. Signal Processing, vol. 47, pp. 1946-1953, July 1999.

[9] A. W. Lohmann, D. Mendlovic, and Z. Zalevsky, "Fractional Hilbert transform," Opt. Lett., vol. 21, pp. 281-283, Feb. 1996.

[10] S. C. Pei and M. H. Yeh, "Discrete fractional Hilbert transform," in Proc. IEEE Int. Symp. Circuits and Systems, vol. 4, May 1998, pp. 506-509.

[11] A. I. Zayed, "Hilbert transform associated with the fractional Fourier transform," IEEE Signal Processing Lett., vol. 5, pp. 206-208, Aug. 1998.

[12] M. Lang and T. I. Laakso, "Simple and robust method for the design of allpass filters using least squares phase error criterion," IEEE Trans. Circuits Syst. II, vol. 41, pp. 40-48, Jan. 1994.

[13] W. S. Lu, S. C. Pei, and C. C. Tseng, "A weighted least-squares method for the design of stable 1-D and 2-D IIR digital filters," IEEE Trans. Signal Processing, vol. 46, pp. 1-10, Jan. 1998.

[14] K. Kohlmann, "Corner detection in natural images based on the 2-D Hilbert transform," Signal Processing, pp. 225-234, 1996.

\section{Roundoff Noise Minimization in a Modified Direct-Form Delta Operator IIR Structure}

\author{
Ngai Wong and Tung-Sang Ng
}

\begin{abstract}
Among various direct-form delta operator realized filter structures, the delta transposed direct-form II ( $\delta$ DFIIt) has been shown to produce the lowest roundoff noise gain in finite wordlength implementations. Recent analyses focus on the optimization of the free parameter $\Delta$ of the delta operator, with scaling of the structure to prevent arithmetic overflow. This paper proposes a modified $\delta$ DFIIt second-order section in which the $\Delta \mathrm{s}$ and filter coefficients at different branches are separately scaled to achieve improved roundoff noise gain minimization. Expressions for the filter coefficients are derived, and reduction of roundoff noise gain is verified by numerical examples.
\end{abstract}

Index Terms-Delta operator, IIR filter, minimization, roundoff noise.

\section{INTRODUCTION}

Delta operator realized filters have attracted increasing attention in this decade due to their good numerical properties when compared to the traditional delay structures [1]-[8]. This is especially true for systems whose sampling rate is much higher than the underlying signal bandwidth, causing the $z$-plane poles to cluster toward the unit circle. By replacing the conventional $z^{-1}$ operator with the inverse delta operator $\delta^{-1}=\Delta z^{-1} /\left(1-z^{-1}\right)$, certain ill-conditioned numerical issues

Manuscript received December 1999; revised October 2000. This work was supported by the Hong Kong Research Grants Council and by the University Research Committee of The University of Hong Kong. This paper was recommended by Associate Editor T. Stathaki.

The authors are with Department of Electrical and Electronic Engineering, The University of Hong Kong, Hong Kong (e-mail: nwong@eee.hku.hk; tsng@eee.hku.hk).

Publisher Item Identifier S 1057-7130(00)11663-5.

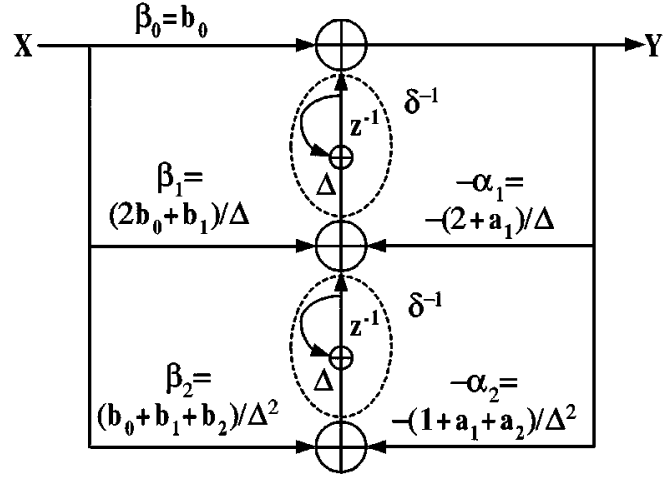

Fig. 1. Delta equivalence of delay structure with transfer function given by (1)

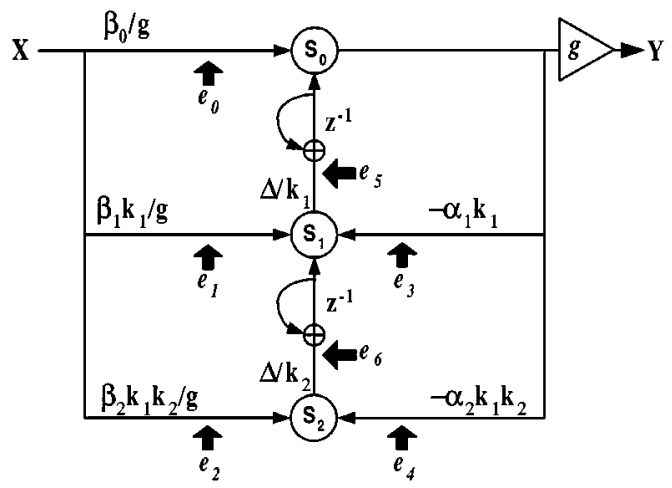

Fig. 2. Modified delta section with overflow scaling by $g$, and $\Delta$ and coefficient scaling by $k_{1}$ and $k_{2}$.

in delay structures can be overcome. In particular, delta operator realizations are generally accompanied with better roundoff noise performance and more robust coefficient and frequency sensitivities [1], [2]. Although the inverse delta operator is more complicated to implement, its excellent numerical properties allow the use of shorter wordlengths, which results in moderate complexity or even gross savings in silicon area [6].

Comprehensive study of different delta structures has been carried out in [4]. It was found that the delta transposed direct-form II ( $\delta$ DFIIt) shows the best roundoff noise properties among various delta structures. Emphasis has been put on the optimization of the free parameter $\Delta(\Delta>0)$ of the delta operator in order to achieve minimum roundoff noise gain at the output. The second-order $\delta$ DFIIt section, being a basic building block, was analyzed in detail [3], [4].

In this paper, instead of limiting to a single optimal $\Delta$ within the second-order $\delta$ DFIIt section, the concept of separately scaling the $\Delta \mathrm{s}$ as well as filter coefficients is introduced. It will be shown that such an approach will enable the true global optimal solution to be obtained, which further minimizes the roundoff noise gain of the section.

\section{NOISE MinimiZATION}

Suppose that a transfer function in the $z$-domain, represented by (1), is obtained under certain specifications and sampling conditions. It can then be transformed into an equivalent delta structure with the substitution $z=1+\delta \Delta$. Fig. 1 shows the $\delta$ DFIIt implementation of (1). This structure was studied extensively for optimization in previous works [3], [4], where the same $\Delta$ was used in both $\delta^{-1}$ operators. Fig. 2 shows 
the proposed equivalent structure, which introduces two additional positive scaling constants $k_{1}$ and $k_{2}$ to the $\Delta \mathrm{s}$ and filter coefficients in the second-order $\delta$ DFIIt section. These scaling constants allow higher degree of freedom for roundoff noise gain minimization.

If a wordlength of less than double precision is used, the summation nodes $S_{0}, S_{1}$, and $S_{2}$ in Fig. 2, known as branch nodes [9], have to be scaled to prevent overflow. The unscaled transfer functions (i.e., $g=1$ ) from the input to these nodes are given in (1)-(3) at the bottom of the page.

A common overflow prevention strategy is to use $L_{\infty}$ norm scaling [9], where the $L_{p}$ norm of a transfer function $F$ is defined as

$$
\|F\|_{p}=\left[\frac{1}{2 \pi} \int_{-\pi}^{\pi}\left|F\left(e^{j \omega}\right)\right|^{p} d \omega\right]^{1 / p} .
$$

Using the convention (also throughout this paper) that a tilde-topped transfer function represents its $z$-dependent part after those prefixing constants, the scaling factor $g$ will be

$$
\begin{aligned}
g & =\max \left(\left\|F_{0}\right\|_{\infty},\left\|F_{1}\right\|_{\infty},\left\|F_{2}\right\|_{\infty}\right) \\
& =\max \left(\left\|F_{0}\right\|_{\infty},\left\|\tilde{F}_{1}\right\|_{\infty} k_{1} \Delta^{-1},\left\|\tilde{F}_{2}\right\|_{\infty} k_{1} k_{2} \Delta^{-2}\right) .
\end{aligned}
$$

The transfer functions from different noise sources to the output as a result of multiplication quantization are given below. It is assumed that noise from the back scaling by $g$ before the output $Y$ is absorbed into the next section

$$
\begin{aligned}
G_{0} & =\frac{Y}{e_{0}}=g \frac{\left(1-z^{-1}\right)^{2}}{1+a_{1} z^{-1}+a_{2} z^{-2}} \\
G_{1,3} & =\frac{Y}{e_{1}}=\frac{Y}{e_{3}}=g \frac{\Delta}{k_{1}} \frac{z^{-1}\left(1-z^{-1}\right)}{1+a_{1} z^{-1}+a_{2} z^{-2}} \\
G_{2,4} & =\frac{Y}{e_{2}}=\frac{Y}{e_{4}}=g \frac{\Delta^{2}}{k_{1} k_{2}} \frac{z^{-2}}{1+a_{1} z^{-1}+a_{2} z^{-2}} \\
G_{5} & =\frac{Y}{e_{5}}=g \frac{z^{-1}\left(1-z^{-1}\right)}{1+a_{1} z^{-1}+a_{2} z^{-2}} \\
G_{6} & =\frac{Y}{e_{6}}=g \frac{\Delta}{k_{1}} \frac{z^{-2}}{1+a_{1} z^{-1}+a_{2} z^{-2}} .
\end{aligned}
$$

Assuming that all products are quantized to the same wordlength by rounding, and modeling rounding quantization error as an uncorrelated white noise process, superposition holds and the output noise variance $\sigma_{o}^{2}$ can be expressed as a sum of noise powers

$$
\begin{gathered}
\sigma_{o}^{2}=\sigma_{e}^{2} g^{2}\left(\left\|\tilde{G}_{0}\right\|_{2}^{2}+\left\|\tilde{G}_{5}\right\|_{2}^{2}+\left(\left\|\tilde{G}_{6}\right\|_{2}^{2}+2\left\|\tilde{G}_{1,3}\right\|_{2}^{2}\right) k_{1}^{-2} \Delta^{2}\right. \\
\left.+2\left\|\tilde{G}_{2,4}\right\|_{2}^{2} k_{1}^{-2} k_{2}^{-2} \Delta^{4}\right)
\end{gathered}
$$

Here $\sigma_{e}^{2}$ stands for the roundoff noise variance of each quantization process and is dependent on the wordlength (or precision) used.

\begin{tabular}{|c|c|c|c|}
\hline$\overline{\Lambda_{o p}^{*} / k_{1}}$ & $\left\|\tilde{F}_{\tilde{F}_{\infty}}\right\|\left\|_{F_{0}}\right\|_{\infty_{0}}$ & $\Delta_{o p}^{*} / k_{2}$ & $\left\|\tilde{F}_{\tilde{r}_{\infty}}\right\|_{\infty} \mid \tilde{F}_{1} \|_{\infty}$ \\
\hline 8 & $\left\|F_{0}\right\|_{\infty}$ & $\beta_{0} / 8$ & $b_{0} /\left\|F_{0}\right\|_{\infty}$ \\
\hline$\overline{\beta_{1} k_{1} / g}$ & $\left(2 b_{0}+b_{1}\right) /\left\|\tilde{F}_{1}\right\|_{\text {。 }}$ & $\beta_{2} k_{1} k_{2} / g$ & $\left(b_{0}+b_{1}+b_{2}\right) / \| \tilde{F}_{2} \mid$ \\
\hline$\alpha_{1} k_{1}$ & $\left(2+a_{1}\right)\left\|F_{0}\right\|\left\|_{\infty} /\right\| \tilde{H}_{\hat{i}} \|$ & $\alpha_{2} k_{1} k_{2}$ & $\left(1+a_{1}+a_{2}\right)\left\|F_{0}\right\|_{0} / \| \tilde{F}_{2} \mid$ \\
\hline
\end{tabular}
A wordlength-independent noise gain term can therefore be defined as the ratio $\sigma_{o}^{2} / \sigma_{e}^{2}$. To minimize this gain, note that the scaling factor is a max-function dependent on $\Delta$ and there are three possible regions of $\Delta$ corresponding to the three possible maxima in (5). Using a similar

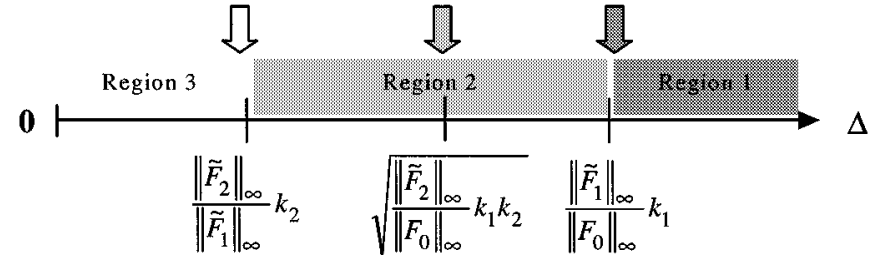

Fig. 3. Optimal $\Delta \mathrm{s}$ in different regions denoted by thick arrows.

TABLE I

VARIOUS EXPRESSIONS FOR THE MODIFIED $\delta$ DFIIt SECOND-ORDER STRUCTURE

approach as in [4], each argument in (5) is set to be the maximum and solved for its valid region. Three regions are obtained, namely

Region 1

$$
\begin{aligned}
& \infty \geq \Delta \geq \max \left(\frac{\left\|\tilde{F}_{1}\right\|_{\infty}}{\left\|F_{0}\right\|_{\infty}} k_{1}, \sqrt{\frac{\left\|\tilde{F}_{2}\right\|_{\infty}}{\left\|F_{0}\right\|_{\infty}} k_{1} k_{2}}\right) \\
& \sigma_{o}^{2} / \sigma_{e}^{2}=\left\|F_{0}\right\|_{\infty}^{2}\left(\left\|\tilde{G}_{0}\right\|_{2}^{2}+\left\|\tilde{G}_{5}\right\|_{2}^{2}\right. \\
&+\left(\left\|\tilde{G}_{6}\right\|_{2}^{2}+2\left\|\tilde{G}_{1,3}\right\|_{2}^{2}\right) k_{1}^{-2} \Delta^{2} \\
&\left.+2\left\|\tilde{G}_{2,4}\right\|_{2}^{2} k_{1}^{-2} k_{2}^{-2} \Delta^{4}\right) .
\end{aligned}
$$

Clearly, as (13) depends only on positive powers of $\Delta$, the output noise gain is minimized by choosing the lower limit of $\Delta$

Region 2

$$
\begin{aligned}
\frac{\left\|\tilde{F}_{1}\right\|_{\infty}}{\left\|F_{0}\right\|_{\infty}} k_{1} \geq \Delta \geq & \frac{\left\|\tilde{F}_{2}\right\|_{\infty} k_{2}}{\left\|\tilde{F}_{1}\right\|_{\infty}} \\
\sigma_{o}^{2} / \sigma_{e}^{2}=\left\|\tilde{F}_{1}\right\|_{\infty}^{2}( & \left(\left\|\tilde{G}_{0}\right\|_{2}^{2}+\left\|\tilde{G}_{5}\right\|_{2}^{2}\right) k_{1}^{2} \Delta^{-2} \\
& +\left(\left\|\tilde{G}_{6}\right\|_{2}^{2}+2\left\|\tilde{G}_{1,3}\right\|_{2}^{2}\right) \\
& \left.+2\left\|\tilde{G}_{2,4}\right\|_{2}^{2} k_{2}^{-2} \Delta^{2}\right) .
\end{aligned}
$$

$$
\begin{aligned}
& F_{0}=\frac{Y}{X}=\frac{S_{0}}{X}=\frac{b_{0}+b_{1} z^{-1}+b_{2} z^{-2}}{1+a_{1} z^{-1}+a_{2} z^{-2}} \\
& F_{1}=\frac{S_{1}}{X}=\frac{k_{1}}{\Delta} \frac{\left(b_{1}-b_{0} a_{1}\right)+\left(b_{0}\left(a_{1}-a_{2}\right)+b_{2}-b_{1}\right) z^{-1}+\left(b_{0} a_{2}-b_{2}\right) z^{-2}}{1+a_{1} z^{-1}+a_{2} z^{-2}} \\
& F_{2}=\frac{S_{2}}{X}=\frac{k_{1} k_{2}}{\Delta^{2}} \frac{\left(b_{1}+b_{2}-b_{0}\left(a_{1}+a_{2}\right)\right)+\left(a_{1}\left(b_{0}+b_{2}\right)-b_{1}\left(1+a_{2}\right)\right) z^{-1}+\left(a_{2}\left(b_{0}+b_{1}\right)-b_{2}\left(1+a_{1}\right)\right) z^{-2}}{1+a_{1} z^{-1}+a_{2} z^{-2}} .
\end{aligned}
$$


TABLE II

NOISE GAIN COMPARISON FOR THE Two EXAMPLE SiXTH-ORDER LOW-PASS FILTERS

\begin{tabular}{|c|c|c|c|c|c|c|}
\hline \multicolumn{7}{|c|}{ SECOND-ORDER SECTIONS FOR FILTERS A AND B } \\
\hline Polynomial & Section A1 & Section A2 & Section $\mathbf{A 3}$ & Section B1 & Section B2 & Section B3 \\
\hline Denominator $\mathbf{a}_{1}, \mathbf{a}_{2}$ & $\begin{array}{c}-1.93504729 \\
0.96471582 \\
\end{array}$ & $\begin{array}{c}-1.86611453 \\
0.88788503 \\
\end{array}$ & $\begin{array}{c}-1.80612859 \\
0.81824041 \\
\end{array}$ & $\begin{array}{r}-1.99512547 \\
0.99610130 \\
\end{array}$ & $\begin{array}{c}-1.98883573 \\
0.98938327 \\
\end{array}$ & $\begin{array}{r}-1.98540165 \\
0.98552386 \\
\end{array}$ \\
\hline Numerator $b_{1}\left(b_{0}=b_{2}=1\right)$ & -1.25901348 & -1.87112896 & -1.92379959 & 2 & 2 & 2 \\
\hline$\sigma_{0}^{2} / \sigma_{e}^{2}$ w.r.t. (18) /dB & 15.0978 & 10.7450 & 8.7220 & 24.1235 & 19.9887 & 19.0695 \\
\hline$\sigma_{0}{ }^{2} / \sigma_{\mathrm{e}}{ }^{2}$ w.r.t. (20) $/ \mathrm{dB}$ & 15.0430 & 9.9691 & 7.0330 & 24.1109 & 19.7033 & 17.4691 \\
\hline Improvement/dB & 0.05483 & 0.7759 & 1.6890 & 0.01263 & 0.2854 & 1.6005 \\
\hline \multicolumn{7}{|c|}{ FLLTER A UNDER VARIOUS CASCADE ORDERINGS } \\
\hline Ordering & A1-A2-A3 & A1-A3-A2 & A2-A1-A3 & A2-A3-A1 & A3-A1-A2 & A3-A2-A1 \\
\hline$\sigma_{0}{ }^{2} / \sigma_{e}{ }^{2}$ w.r.t. (18) $/ \mathrm{dB}$ & 21.3401 & 19.9110 & 18.4540 & 17.6521 & 17.2400 & 19.1439 \\
\hline$\sigma_{0}{ }^{2} / \sigma_{\mathrm{e}}{ }^{2}$ w.r.t. (20) $/ \mathrm{dB}$ & 21.0604 & 19.7857 & 18.1802 & 16.9020 & 16.5724 & 18.3415 \\
\hline Improvement /dB & 0.2798 & 0.1253 & 0.2737 & 0.7501 & 0.6676 & 0.8025 \\
\hline \multicolumn{7}{|c|}{ FILTER B UNDER VARIOUS CASCADE ORDERINGS } \\
\hline Ordering & B1-B2-B3 & B1-B3-B2 & B2-B1-B3 & B2-B3-B1 & B3-B1-B2 & B3-B2-B1 \\
\hline$\sigma_{0}{ }^{2} / \sigma_{e}{ }^{2}$ w.r.t. $(18) / \mathrm{dB}$ & 33.9075 & 31.4075 & 30.0289 & 28.2737 & 28.6666 & 30.5646 \\
\hline$\sigma_{0}{ }^{2} / \sigma_{\mathrm{e}}{ }^{2}$ w.r.t. (20) $/ \mathrm{dB}$ & 33.7262 & 31.2584 & 29.7870 & 27.7263 & 28.2592 & 30.0011 \\
\hline Improvement /dB & 0.1813 & 0.1491 & 0.2419 & 0.5474 & 0.4074 & 0.5634 \\
\hline
\end{tabular}

As (15) depends on both positive and negative powers of $\Delta$, the $\Delta$ that minimizes (15) is expected to lie somewhere in between the limits Region 3

$\min \left(\sqrt{\frac{\left\|\tilde{F}_{2}\right\|_{\infty}}{\left\|F_{0}\right\|_{\infty}} k_{1} k_{2}}, \frac{\left\|\tilde{F}_{2}\right\|_{\infty}}{\left\|\tilde{F}_{1}\right\|_{\infty}} k_{2}\right) \geq \Delta \geq 0$

and

$$
\begin{aligned}
\sigma_{o}^{2} / \sigma_{e}^{2}=\left\|\tilde{F}_{2}\right\|_{\infty}^{2} & \left(\left(\left\|\tilde{G}_{0}\right\|_{2}^{2}+\left\|\tilde{G}_{5}\right\|_{2}^{2}\right) k_{1}^{2} k_{2}^{2} \Delta^{-4}\right. \\
& +\left(\left\|\tilde{G}_{6}\right\|_{2}^{2}+2\left\|\tilde{G}_{1,3}\right\|_{2}^{2}\right) k_{2}^{2} \Delta^{-2} \\
& \left.+2\left\|\tilde{G}_{2,4}\right\|_{2}^{2}\right) .
\end{aligned}
$$

Since (17) depends only on negative powers of $\Delta$, the output noise gain is minimized by setting $\Delta$ equal to the upper limit.

Fig. 3 shows a possible partition of these regions. The thick arrows indicate possible choices of the local optimal $\Delta \mathrm{s}$ according to the previous reasoning. Past analyses [3], [4] implicitly set $k_{1}=k_{2}=1$, and a single optimal $\Delta$ is chosen for the whole second-order section by comparing the noise gain minima in different regions. In [4], this optimal $\Delta$ is given by

$$
\Delta_{o p}=\max \left(\frac{\left\|\tilde{F}_{1}\right\|_{\infty}}{\left\|F_{0}\right\|_{\infty}}, \sqrt{\frac{\left\|\tilde{F}_{2}\right\|_{\infty}}{\left\|F_{0}\right\|_{\infty}}}\right) .
$$

However, in the proposed section in this paper, $k_{1}$ and $k_{2}$ are chosen such that

$$
\frac{\left\|\tilde{F}_{1}\right\|_{\infty}}{\left\|F_{0}\right\|_{\infty}} k_{1}=\frac{\left\|\tilde{F}_{2}\right\|_{\infty}}{\left\|\tilde{F}_{1}\right\|_{\infty}} k_{2}=\sqrt{\frac{\left\|\tilde{F}_{2}\right\|_{\infty}}{\left\|F_{0}\right\|_{\infty}} k_{1} k_{2}} .
$$

The third term in (19) is in fact the geometric mean of the first two terms. Now by substituting them into (12), (14), and (16), it is apparent that the three local minima in the three different regions converge to the same point. Hence the global optimal $\Delta$ (denoted by an asterisk) is given by

$$
\Delta_{\text {op }}^{*}=\frac{\left\|\tilde{F}_{1}\right\|_{\infty}}{\left\|F_{0}\right\|_{\infty}} k_{1}=\frac{\left\|\tilde{F}_{2}\right\|_{\infty}}{\left\|\tilde{F}_{1}\right\|_{\infty}} k_{2}
$$

From (20), the ratios $\Delta_{\mathrm{op}}^{*} / k_{1}$ and $\Delta_{\mathrm{op}}^{*} / k_{2}$ can be obtained since $\left\|F_{0}\right\|_{\infty},\left\|\tilde{F}_{1}\right\|_{\infty},\left\|\tilde{F}_{2}\right\|_{\infty}$ are computable. Consequently, the global optimal $\Delta$ becomes implicit and is embedded into the ratios. Using these results, neat expressions for each filter coefficient and the noise variance of the proposed $\delta$ DFIIt section (Fig. 2) can be derived and are given in Table I.

An interesting observation is that all the node norms in the scaling factor (5) are now equal to the filter norm, i.e., $\left\|F_{0}\right\|_{\infty}$. Usually the filter norm is designed to utilize the dynamic range of the finite wordlength. Therefore, by equalizing all node norms to the filter norm, the dynamic range at all branch nodes is utilized. In an efficient hardware implementation, the scaled $\Delta \mathrm{s}$ can be rounded to the nearest powers of two so that multiplication can be accomplished by simple bit shifting.

\section{NUMERICAL EXAMPLES}

Several second-order sections are taken from [4] to test against the proposed approach. Sections $A i$ and $B i, i=1,2,3$, as shown in Table II, are cascade sections for two example sixth-order narrow-band low-pass filters. For fair comparison, and to eliminate the effect of word precision on the output noise variance, the wordlength-independent noise gain term defined as $\sigma_{o}^{2} / \sigma_{e}^{2}$ [see (11)] is used. First, each section is individually prescaled using its $L_{\infty}$ norm, with scaling embedded into the numerator coefficients. The noise gain of each section is then evaluated with respect to the $\Delta_{\mathrm{op}}$ in (18) and the proposed $\Delta_{\mathrm{op}}^{*}$ in (20). It is apparent that there is always reduction in roundoff noise gain using the proposed approach, especially when the pole angle decreases. Next, each of the three sections is cascaded to investigate the effect of different section orderings. Along the cascade system of each ordering, $L_{\infty}$ norm scaling is distributed and embedded into the numerator coefficients such that overflow is prevented at the output of every second-order section [4]. Again, as shown in Table II, there is a 
consistent reduction in roundoff noise gain in whichever order the sections are cascaded.

\section{CONCLUSION}

In this paper, the concept of separately scaling the $\Delta \mathrm{s}$ and filter coefficients in the $\delta$ DFIIt section has been introduced. This approach leads to further improved global minimization of output roundoff noise gain as compared to choosing a single optimal $\Delta$ only. Using numerical examples, roundoff noise performance of this modified $\delta$ DFIIt second-order structure has been demonstrated to be better than the best results obtained so far. Readily computable expressions for the optimal filter coefficients have also been derived.

\section{REFERENCES}

[1] R. H. Middleton and G. C. Goodwin, Digital Control and Estimation: A Unified Approach. Englewood Cliffs, NJ: Prentice Hall, 1990.

[2] G. C. Goodwin, R. H. Middleton, and H. V. Poor, "High speed digital signal processing and control," Proc. IEEE, vol. 80, pp. 240-259, Feb. 1992.

[3] J. Kauraniemi, T. I. Laakso, I. Hartimo, and S. J. Ovaska, "Roundoff noise minimization in a direct form delta operator structure," in Proc. 1996 Int. Conf. Acoustics, Speech, and Signal Processing, Atlanta, GA, May 1996.

[4] J. Kauraniemi, T. I. Laakso, I. Hartimo, and S. J. Ovaska, "Delta operator realizations of direct-form IIR filters," IEEE Trans. Circuits Syst. II, vol. 45, pp. 41-52, Jan 1998.

[5] J. Kauraniemi and T. I. Laakso, "Roundoff noise analysis of modified delta operator direct form structures," in Proc. IEEE Int. Symp. Circuits Systems, 1997.

[6] M. Eraluoto, J. Kautraniemi, and I. Hartimo, "VLSI implementation of high speed digital filters using direct form delta structures," in Proc. IEEE 39th Midwest Symp. Circuits Systems, 1996.

[7] G. Li and M. Gevers, "Roundoff noise minimization using delta operato realizations," IEEE Trans. Signal Processing, vol. 41, pp. 629-637, Feb. 1993.

[8] G. Li and M. Gevers, "Comparative study of finite wordlength effects in shift and delta operator parameterizations," IEEE Trans. Automat. Contr., vol. 38, pp. 803-807, May 1993.

[9] L. B. Jackson, Digital Filters and Signal Processing, 2nd ed. Boston, MA: Kluwer Academic , 1989.

\section{Approximation of Classical IIR Filters with Additional Specifications}

\author{
H. H. Dam, S. Norbedo, and L. Svensson
}

\begin{abstract}
In this paper, we introduce a modified tapped all-pass (MTA) structure for the sum of two all-pass functions. The new structure includes linear coefficients in the all-pass sections, allowing additional constraints such as null or group delay constraints for the filters. These null constraints are significant for applications when the suppression of the noise at certain frequencies is important.

An alternative structure to incorporate the extra constraints is the modified tapped cascade (MTC) infinite impulse response structure. Design examples show that the MTA structure is less sensitive to quantization than the MTC structure in the passband. Moreover, the total number of multiplications is significantly reduced by using the MTA structure as compared to the MTC structure.
\end{abstract}

Index Terms-Digital filter made as the sum of all-pass functions, MTA, MTC, null constraints.

\section{INTRODUCTION}

It is straightforward to design filter transfer functions with Butterworth, Chebyshev, or elliptic characteristics. The placement of poles and zeros, which guarantee certain limits on the passband and stopband attenuation, are available in tabulated or algebraic form given the filter order, passband and stopband ripples, and transition bandwidth. However, the transfer functions are based on the underlying assumption that the power density of the rejected signal is constant across the stopband, or at least the designer does not have any information about it. The frequencies of the high-attenuation dips, therefore, do not matter. In actual design problems, some information about the power density is usually available, but this information cannot be included in the design of classical filters.

In this paper, the design of a parallel all-pass filter structure with linear coefficients, as shown in Fig. 1, is investigated. This modified tapped all-pass (MTA) filter structure is a generalization of the Laguerre structure [1]. The filter structure is obtained from the sum of two all-pass functions by imposing a set of linear coefficients in the all-pass sections. These linear coefficients allow additional filter specifications such as null or group delay constraints. Additional null constraints will be discussed in this paper. These null constraints are significant for applications when the suppression of the noise at certain frequencies is important. The implementation of group delay constraints will be similar.

The transfer functions from the filter inputs to the all-pass section outputs constitute basis functions for optimization of the linear coefficients. One could consider an optimal design that includes selecting the associated basis functions and the linear coefficients. This optimization

Manuscript received May 2000. This work was supported by the Department of Telecommunications and Signal Processing, University of Karlskrona/Ronneby, Sweden. This paper was receommended by Associate Editor V. Anastassopoulos.

H. H. Dam is with the Australian Telecommunications Research Institute, Curtin University of Technology, Perth 6845 Australia (e-mail: dam@atri.curtin.edu.au).

S. Norbedo is with the Department of Telecommunications and Signal Processing, University of Karlskrona/Ronneby, Ronneby S-372 25 Sweden (e-mail sven.nordebo@its.hk-r.se).

L. Svensson was with Ericsson Mobile Communications, SE-221 83 Lund, Sweden. He is now with SwitchCore AB, SE-411 04 Göteborg, Sweden (e-mail lars.svensson@switchcore.com).

Publisher Item Identifier S 1057-7130(00)11654-4. 